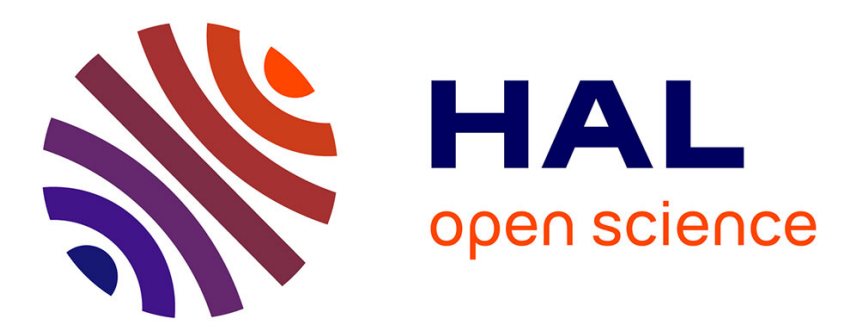

\title{
Ordre-désordre des lacunes anioniques dans les perovskites non stoechiométriques CaTi1-2yFe2yO3-y
}

Jean-Claude Grenier, M. Pouchard, Paul Hagenmuller, Gérard Schiffmacher, Paul Caro

\section{- To cite this version:}

Jean-Claude Grenier, M. Pouchard, Paul Hagenmuller, Gérard Schiffmacher, Paul Caro. Ordredésordre des lacunes anioniques dans les perovskites non stoechiométriques CaTi1-2yFe2yO3-y. Journal de Physique Colloques, 1977, 38 (C7), pp.84-90. 10.1051/jphyscol:1977717 . jpa-00217218

\section{HAL Id: jpa-00217218 https://hal.science/jpa-00217218}

Submitted on 1 Jan 1977

HAL is a multi-disciplinary open access archive for the deposit and dissemination of scientific research documents, whether they are published or not. The documents may come from teaching and research institutions in France or abroad, or from public or private research centers.
L'archive ouverte pluridisciplinaire HAL, est destinée au dépôt et à la diffusion de documents scientifiques de niveau recherche, publiés ou non, émanant des établissements d'enseignement et de recherche français ou étrangers, des laboratoires publics ou privés. 


\title{
ORDRE-DÉSORDRE DES LACUNES ANIONIQUES DANS LES PEROVSKITES NON STCEHIOMETRIQUES $\mathrm{CaTi}_{1-2 y} \mathrm{Fe}_{2 y} \mathrm{O}_{3-y}$
}

\author{
J. C. GRENIER, M. POUCHARD, P. HAGENMULLER
}

Laboratoire de Chimie du Solide du C.N.R.S., Université de Bordeaux I, 351, cours de la Libération, 33405 Talence Cedex, France

\section{G. SCHIFFMACHER et P. CARO}

\author{
Laboratoire des Terres Rares, C.N.R.S., 1, place Aristide-Briand, 92190 Meudon Bellevue, France
}

\begin{abstract}
Résumé. - Un modèle sur la nature de la non-stochiométrie dans les perovskites lacunaires $\mathrm{A}_{n} \mathrm{M}_{n} \mathrm{O}_{3 n-1}(n \geqslant 2)$ a été proposé et vérifié lors de l'étude de la solution solide $\mathrm{CaTi}_{1-2 y} \mathrm{Fe}_{2 y} \mathrm{O}_{3-y}$ $(0 \leqslant y \leqslant 0,50)$ par diffraction $X$, microscopie électronique et spectroscopie Mössbauer. Pour $y \geqslant 0,25(2 \leqslant n \leqslant 4)$ les lacunes s'ordonnent dans des plans $(0 k 0)$ en rangées parallèles à la direction [101] du réseau perovskite. Des composés définis correspondant aux termes $n=2 ; 2,5 ; 3$ et 4 ont été identifiés. Pour des valeurs intermédiaires de $n$, des phénomènes de syntaxie ont été observés. La structure de ces phases est une succession de plans d'octaèdres liés entre eux par un feuillet de tétraèdres. La symétrie est orthorhombique. Pour $y<0,25$, les lacunes anioniques semblent être distribuées statistiquement et la symétrie est pseudocubique. En fait, une étude par spectroscopie Mössbauer révèle la présence de fer trivalent au sein de tétraèdres même pour de faibles taux en lacunes. Ces résultats sont discutés.
\end{abstract}

\begin{abstract}
A structural model for the non-stoichiometric perovskites $\mathrm{A}_{n} \mathrm{M}_{n} \mathrm{O}_{3 n-1}(n \geqslant 2)$ has been proposed and checked by X-ray diffraction, electron microscopy and Mössbauer spectroscopy studies of the $\mathrm{CaTi}_{1-2 y} \mathrm{Fe}_{2 y} \mathrm{O}_{3-y}(0 \leqslant y \leqslant 0.50)$ solid solution.

For $y \geqslant 0.25(2 \leqslant n \leqslant 4)$, the oxygen vacancies are ordered in the $(0 k 0)$ planes in strings parallel to the [101] direction of the perovskite cell. New compounds corresponding to the $n=2 ; 2.5 ; 3$ and 4 terms have been identified. For intermediary values of $n$, phenomena of intergrowth have been observed. The structure of these phases is a succession of sheets of octahedra separated by a sheet of tetrahedra. The symmetry is orthorhombic. For $y<0.25$, anionic vacancies seem to be randomly distributed and the symmetry is pseudocubic. In fact, a Mössbauer study shows the existence of trivalent iron in tetrahedra even for small concentrations of vacancies. Theses results are discussed.
\end{abstract}

De nombreux travaux ont montré que la perte d'oxygène dans les oxydes binaires tels que $\mathrm{TiO}_{2}$, $\mathrm{WO}_{3}$ ou $\mathrm{MoO}_{3}$, conduisait à une compression de la structure initiale en faisant intervenir des mécanismes de cisaillement. Ces phénomènes ont pu être confirmés par des observations directes en microscopie électronique $(1,2)$. Très peu d'études, en revanche, ont traité de l'ordre des lacunes au sein des oxydes ternaires $\mathrm{AMO}_{3-y}$ dérivés de la perovskite (3). L'existence d'un rapport constant entre les cations $A$ et $\mathrm{M}(\mathrm{A} / \mathrm{M}=1)$ lorsque le taux en lacunes évolue $(0 \leqslant y \leqslant 0,50)$ interdit l'apparition de plans de cisaillement analogues à ceux observés dans les oxydes non stæchiométriques $\mathrm{WO}_{3-x}$ dont la structure est voisine. Ceci nous a amené à proposer un nouveau type de non-stœchiométrie pour ces phases (4).

Afin de vérifier notre modèle, nous avons entrepris une étude complète par diffraction $\mathrm{X}$, diffraction et microscopie électronique et spectroscopie Mössbauer des phases $\mathrm{CaTi}_{1-2 y} \mathrm{Fe}_{2 y} \mathrm{O}_{3-y}, 0 \leqslant y \leqslant 0,50$. L'apparition d'une lacune d'oxygène est assurée par le remplacement de deux atomes de titane par deux atomes de fer de rayons ioniques proches

$$
\left(r_{\mathrm{Ti}^{4+}}=0,605 \AA, r_{\mathrm{Fe}^{3+}}=0,645 \AA\right)
$$

selon l'égalité :

$$
2 \mathrm{Ti}^{4+}+\mathrm{O}^{2-}=2 \mathrm{Fe}^{3+}+\square_{\mathrm{O}^{2-}} .
$$

Les perovskites lacunaires $A_{n} M_{n} \mathbf{O}_{3 n-1}$ : hypothèses structurales. - Le modèle structural proposé a été suggéré par une description du ferrite bicalcique $\mathrm{Ca}_{2} \mathrm{Fe}_{2} \mathrm{O}_{5}$ considéré comme une perovskite lacunaire en oxygène et correspondant à $y=0,50$, soit $\mathrm{AMO}_{2,50}$. Ce modèle suppose que les lacunes anioniques s'ordonnent selon certaines rangées [101] de la maille cubique de la perovskite (figure 1). Il se produit un réarrangement local avec formation de files de tétraèdres à sommets communs qui s'ordonnent 


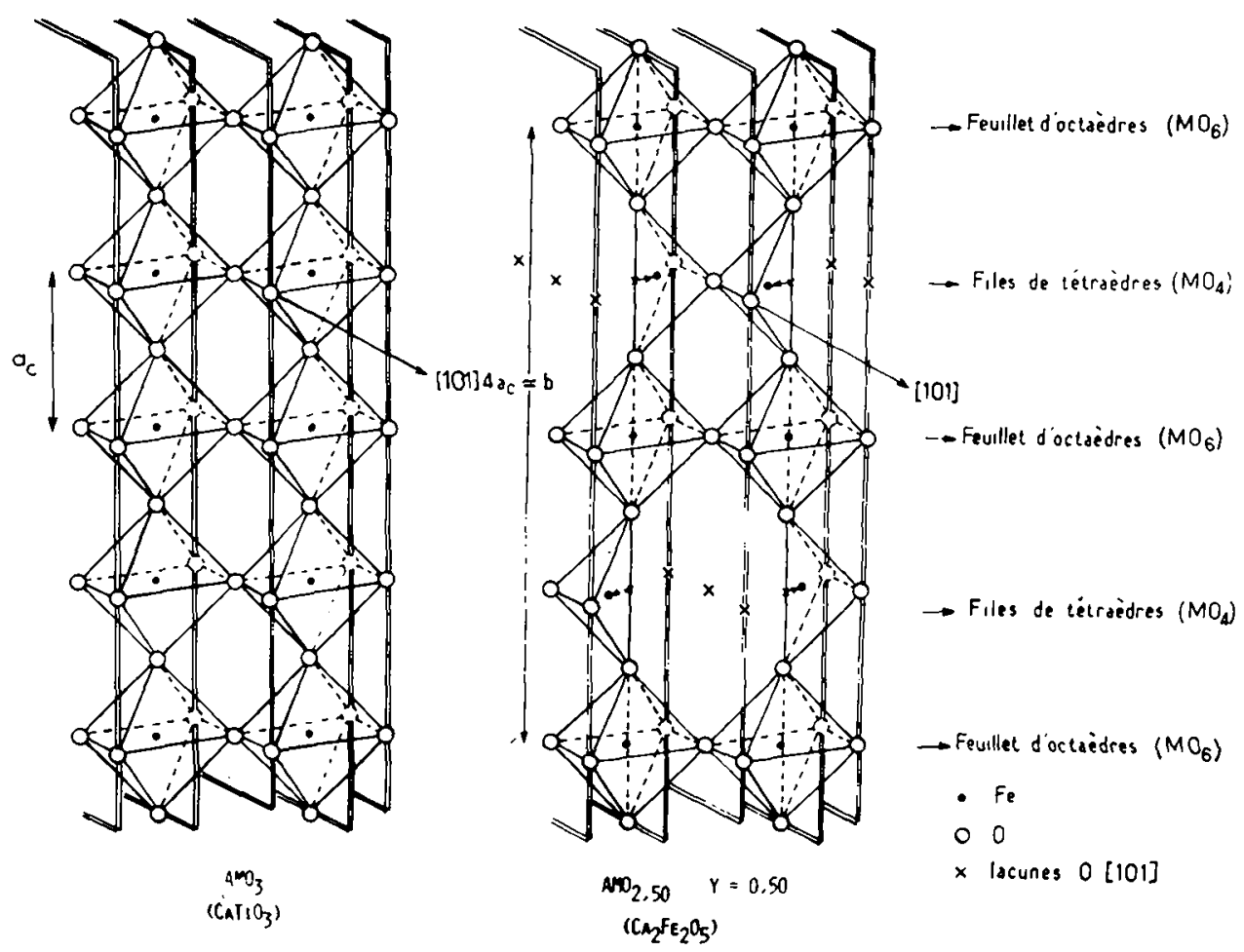

Fig. 1. - Structures idéalisées de la perovskite $\mathrm{CaTiO}_{3}$ et du ferrite bicalcique $\mathrm{Ca}_{2} \mathrm{Fe}_{2} \mathrm{O}_{5}$.

à leur tour, dans des plans $(0 k 0)$. Le réseau de $\mathrm{Ca}_{2} \mathrm{Fe}_{2} \mathrm{O}_{5}$ se forme alors par l'alternance perpendiculairement à l'axe $O y$ de feuillets d'octaèdres et de plans formés de files de tétraèdres.

Dans cette hypothèse, l'apparition périodique dans la structure perovskite de plans de tétraèdres perpendiculaires à l'axe $O y$ pouvait conduire à la formation de nouvelles phases intermédiaires entre les structures de $\mathrm{Ca}_{2} \mathrm{Fe}_{2} \mathrm{O}_{5}$ et de $\mathrm{CaTiO}_{3}$. Ces phases se formuleraient $\mathrm{A}_{n} \mathrm{M}_{n} \mathrm{O}_{3 n-1}(n>2, y=1 / n)$. Leur structure serait un empilement de $n-1$ feuillets successifs d'octaèdres liés entre eux par un feuillet de tétraèdres. La figure 2 représente de manière idéalisée les termes $n=2,3,4$ et $\infty$ des phases $\mathrm{A}_{n} \mathrm{M}_{n} \mathrm{O}_{3 n-1}$. En supposant une symétrie orthorhombique, les paramètres théoriques se déduisent du paramètre $a_{c}$ de la maille cubique de la perovskite $(n=\infty)$ par les relations :

$$
a_{n} \simeq \sqrt{2} a_{\mathrm{c}} \quad b_{n} \simeq n a_{\mathrm{c}} \text { et } \quad c_{n} \simeq \sqrt{2} a_{\mathrm{c}} .
$$

Synthèse des échantillons : analyse radiocristallographique. - Les échantillons sont préparés à partir du carbonate de calcium et des oxydes de fer et de titane selon le schéma réactionnel suivant :

$$
\begin{aligned}
\mathrm{CaCO}_{3}+(1-2 y) \mathrm{TiO}_{2}+y \mathrm{Fe}_{2} \mathrm{O}_{3} \rightarrow \\
\rightarrow \mathrm{CaTi}_{1-2 y} \mathrm{Fe}_{2 y} \mathrm{O}_{3-y}+\widehat{\mathrm{CO}}_{2} .
\end{aligned}
$$

Après un préchauffage à $1100^{\circ} \mathrm{C}$, la réaction s'achève à $1350^{\circ} \mathrm{C}$. Un recuit de deux semaines à
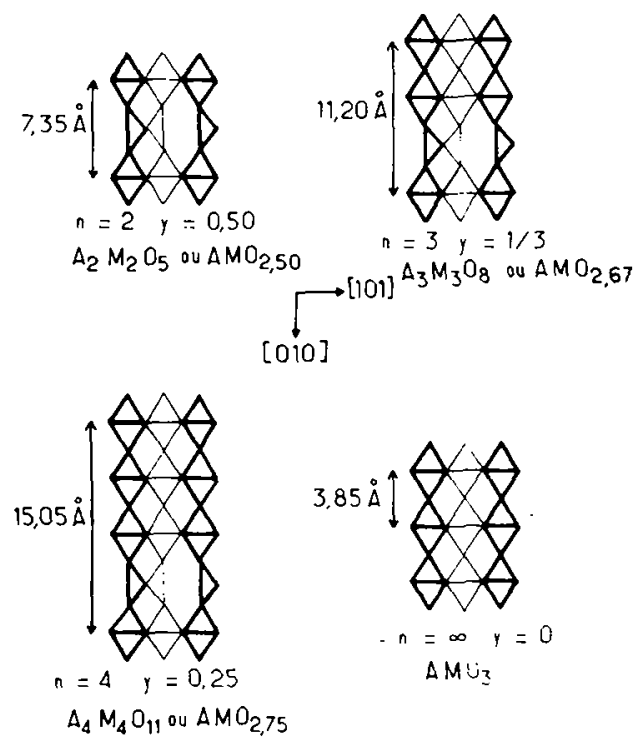

$n=\infty \quad y=0$

$A M U_{3}$

FIg. 2. - Ordonnancement idéalisé des octaèdres et des tétraèdres oxygénés au scin des phases $A_{n} \mathrm{M}_{n} \mathrm{O}_{3 n-1}$.

$1100^{\circ} \mathrm{C}$ sous argon purifié permet d'obtenir un produit homogène, exempt de fer tétravalent. La pression partielle d'oxygène dans l'enceinte réactionnelle, mesurée à l'aide d'une pile de concentration à zircone stabilisée $\left(0,85 \mathrm{ZrO}_{2}, 0,15 \mathrm{CaO}\right)$, est voisine de $3 \times 10^{-4}$ atm., domaine de pression où seul le fer trivalent est stable.

L'analyse des phases obtenues par diffraction $\mathrm{X}$ révèle une évolution continue de la solution solide 
avec $y$. Aucun domaine biphasé n'apparaît mais deux comportements différents des lacunes sont mis en évidence selon les valeurs de $y$.

Pour des valeurs proches de $\mathrm{CaTiO}_{3}(y<0,25)$, la symétrie pseudocubique de la perovskite est conservée; les lacunes anioniques semblent désordonnées.

En revanche pour des compositions proches de $\mathrm{Ca}_{2} \mathrm{Fe}_{2} \mathrm{O}_{5}(y \geqslant 0,25)$ un ordre lacunaire bien déterminé semble apparaître. Les spectres $\mathrm{X}$ de ces phases s'indexent dans le système orthorhombique avec les paramètres prévus par notre modèle.

Le passage d'un état désordonné à un état ordonné s'effectue assez brutalement au voisinage de $y=0,25$.

Ce phénomène est traduit clairement à la figure 3 par une discontinuité dans l'évolution avec $y$ du paramètre moyen $\bar{a}_{y}$ mesuré suivant $O y$. Pour les phases pseudocubiques, $\bar{a}_{y}$ est la moyenne arithmétique des paramètres de la maille pseudocubique; pour les phases orthorhombiques $\bar{a}_{y}$ est calculé à partir du paramètre $b_{n}$ par la relation $\bar{a}_{y}=b_{n} / n(6)$.

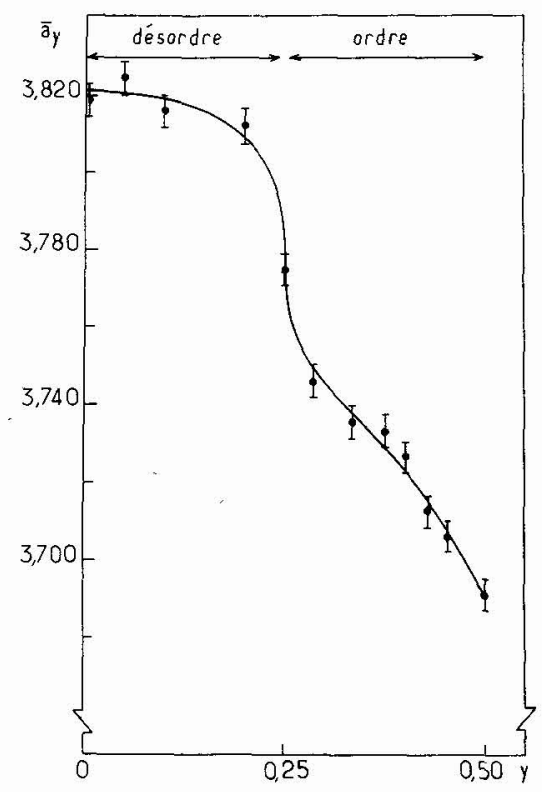

FIG. 3. - Evolution avec $y$ du paramètre moyen $\bar{a}_{y}$.

Microscopie électronique : résultats expérimentaux. - La succession, telle que le prévoit notre modèle, de plans d'octaèdres et de tétraèdres dont la densité électronique est différente, devait se traduire sur les clichés de microscopie électronique par une image de réseau en corrélation directe avec les modèles structuraux.

L'examen au moyen d'un microscope électronique à haute résolution des échantillons précédents a permis de compléter l'étude par diffraction $X$.

Nous avons ainsi vérifié que pour $y \geqslant 0,25$, les lacunes s'ordonnaient dans des plans pour constituer des files de tétraèdres. Nous avons mis en évidence les termes:

$$
\begin{aligned}
& -n=2, y=0,50: \mathrm{CaFeO}_{2,50} \text { ou } \mathrm{Ca}_{2} \mathrm{Fe}_{2} \mathrm{O}_{5} \\
& -n=3, y=0,33: \mathrm{CaTi}_{0,33} \mathrm{Fe}_{0,67} \mathrm{O}_{2,67} \text { ou } \\
& \mathrm{Ca}_{3} \mathrm{Fe}_{2} \mathrm{TiO}_{8} \\
& -n=4, \quad y=0,25 \quad: \quad \mathrm{CaTi}_{0,50} \mathrm{Fe}_{0,50} \mathrm{O}_{2,75} \text { ou } \\
& \mathrm{Ca}_{4} \mathrm{Fe}_{2} \mathrm{Ti}_{2} \mathrm{O}_{11} \text {. }
\end{aligned}
$$

Pour ces composés, l'image de réseau obtenue perpendiculairement à l'axe $O y$ révèle un ordre à grande distance et l'interfrange mesurée est caractéristique de la distance entre deux plans de défauts (plans de tétraèdres (figures 4,5 et 6 )). Ces résultats constituent une première vérification de notre modèle structural.

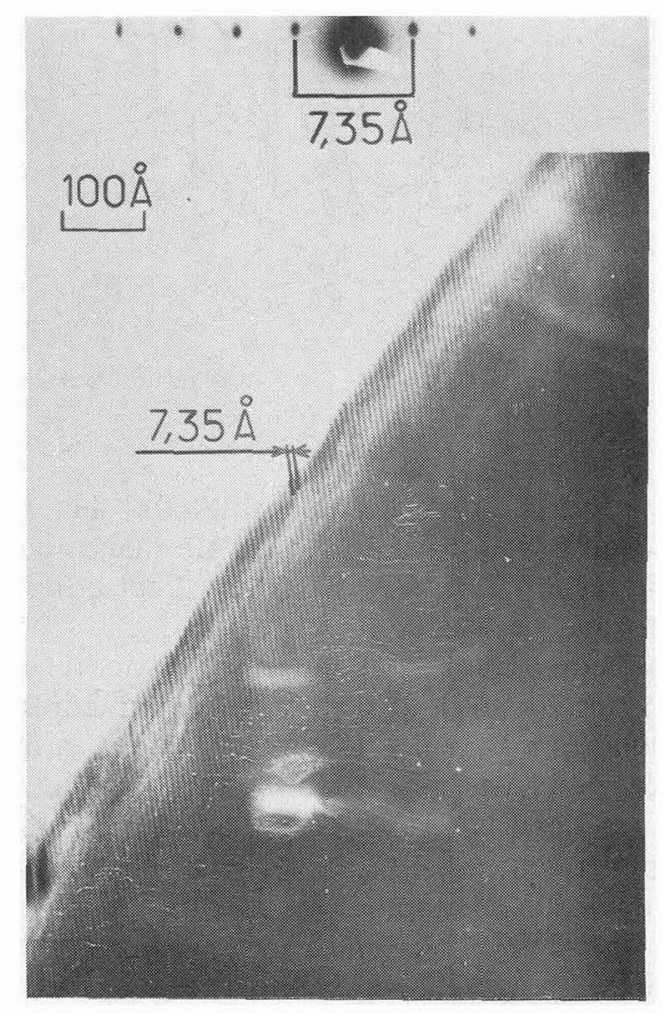

Fig. 4. - Diagramme de diffraction électronique et image de réseau de $\mathrm{Ca}_{2} \mathrm{Fe}_{2} \mathrm{O}_{5}(n=2, y=0,50)$ perpendiculairement à l'axe $O y$.

Pour les phases correspondant à des valeurs intermédiaires de $n, 2<n<3$ soit $0,33<y<0,50$, la diffraction $\mathrm{X}$ montrait une évolution continue des paramètres. Nous avons observé microscopiquement ce que signifiait le passage progressif de $n=2$ à $n=3$. L'examen de $\mathrm{Ca}_{5} \mathrm{Fe}_{4} \mathrm{TiO}_{13}(n=2,5)$ correspondant à la composition $y=0,40$, révèle une structure cristalline très largement ordonnée (figure 7). Contrairement aux images précédentes, les franges obtenues ne sont pas équidistantes mais il apparaît une périodicité voisine de $18,6 \AA$ (espacement $D$ sur la figure 7). Cette périodicité peut être facilement reliée à la structure hypothétique de $\mathrm{Ca}_{5} \mathrm{Fe}_{4} \mathrm{TiO}_{13}$ (figure 8a) qui correspondrait à une succession ordon- 


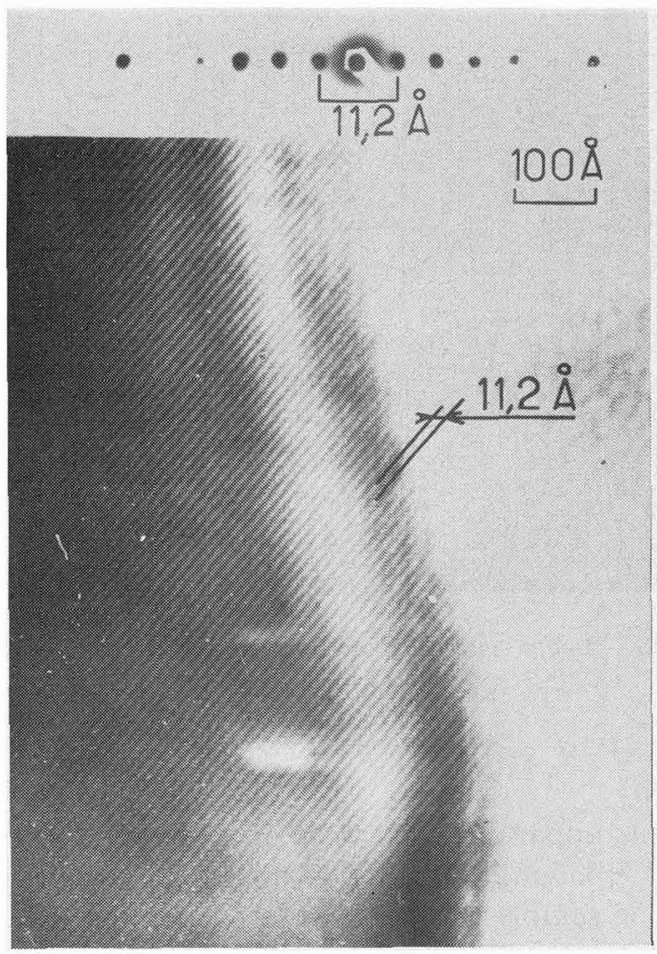

FIG. 5. - Diagramme de diffraction électronique et image de réseau de $\mathrm{Ca}_{3} \mathrm{Fe}_{2} \mathrm{TiO}_{8}(n=3, y=1 / 3)$ perpendiculairement à l'axe $O y$.

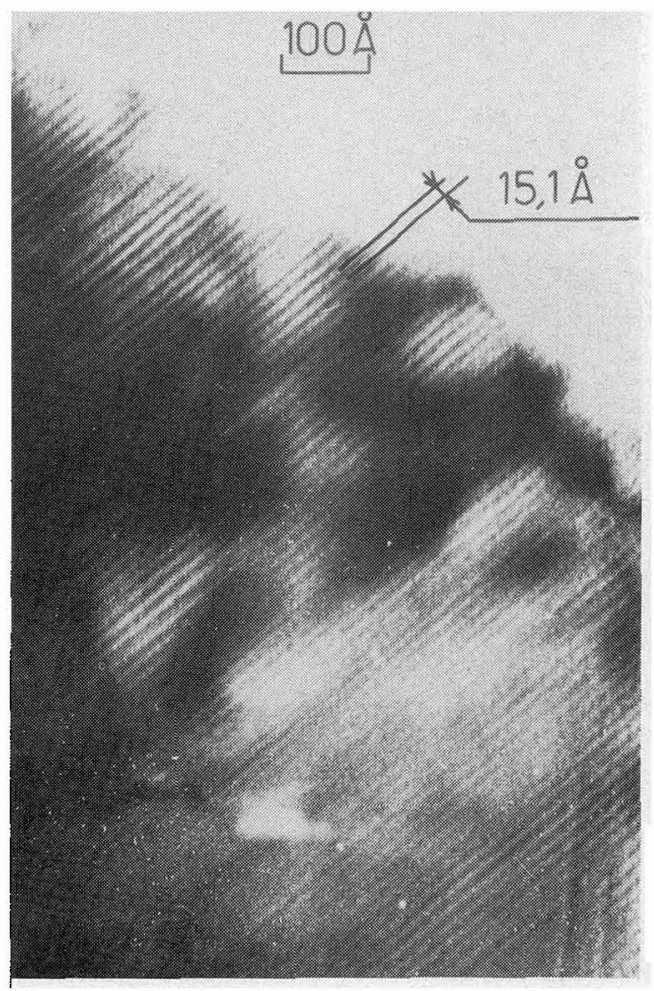

FIG. 6. - Image microscopique de réseau d'un domaine correspondant à $n=4$ de $\mathrm{Ca}_{4} \mathrm{Fe}_{2} \mathrm{Ti}_{2} \mathrm{O}_{11}(y=0,25)$.

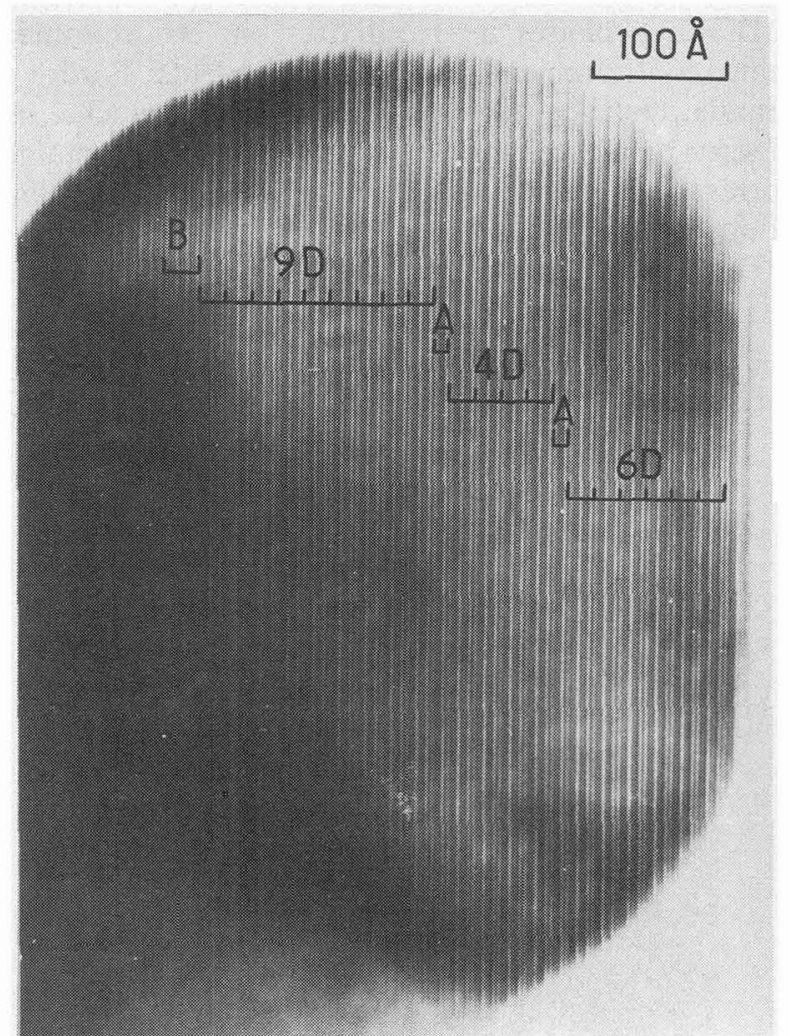

Fig. 7. - Image microscopique de réseau de $\mathrm{Ca}_{5} \mathrm{Fe}_{4} \mathrm{TiO}_{13}$ $(n=2,50 ; y=0 ; 40)$ avec $D \simeq 18,6 \AA$.

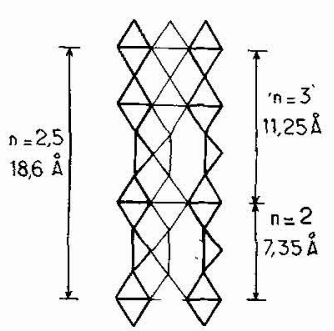

(a)

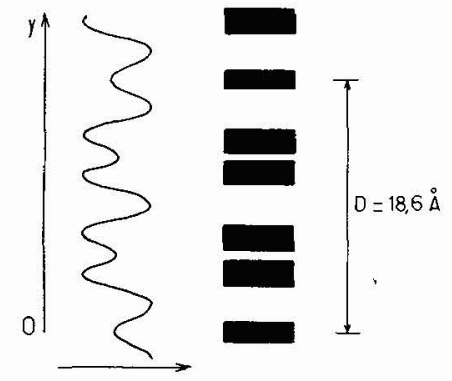

(b)
Frg. 8. - (a) Structure idéalisée de $\mathrm{Ca}_{5} \mathrm{Fe}_{4} \mathrm{TiO}_{13}(n=2,5)$; (b) Variation schématique de la densité électronique suivant $O y$ et des franges correspondantes.

née des termes $n=2$ et $n=3$. La figure $8 b$ représente la variation schématique de la densité électronique suivant $O y$ et les franges qui, selon cette hypothèse, peuvent lui être associées. Dans le cristal observé, il apparaît toutefois deux défauts de périodicité. Le défaut de type $\mathrm{A}$ correspond à l'absence d'un motif $n=2$, alors que le défaut de type $B$ est dû à la présence d'un motif $n=2$ supplémentaire. On peut raisonnablement penser que ces défauts se répartissent statistiquement au sein des cristaux de manière à respecter globalement la composition nominale. 
D'autres phases intermédiaires ont été examinées mais contrairement aux cas précédents l'ordre à grande distance n'apparaît pas. En revanche, on observe une succession désordonnée de microdomaines correspondant à des valeurs de $n$ proches de la valeur nominale.

Deux exemples illustrent ceci :

- pour $\mathrm{Ca}_{7} \mathrm{Fe}_{6} \mathrm{TiO}_{18}(n=2,33 ; y=0,43)$, trois microdomaines différents ont été identifiés (figure 9). Ce sont $D \simeq 18,6 \AA(n=2,50), E \simeq 26 \AA(n=2,33)$ et $F \simeq 33 \AA(n=2,25)$;

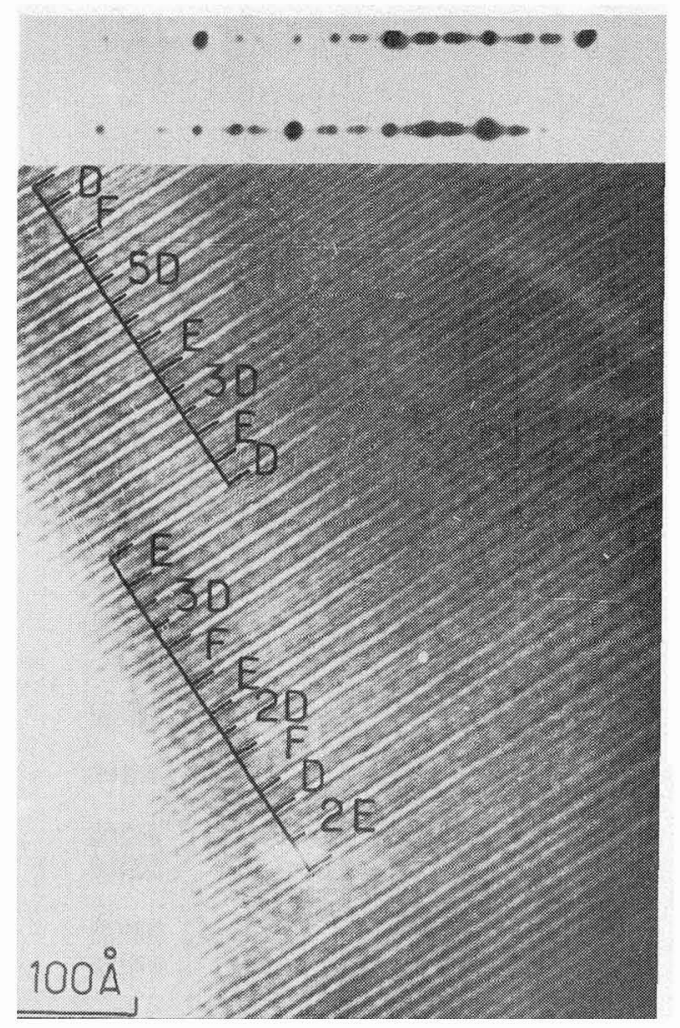

Frg. 9. - Diagramme de diffraction électronique et image de réseau de $\mathrm{Ca}_{7} \mathrm{Fe}_{6} \mathrm{TiO}_{18}(n=2,33 ; y=0,43)$ avec $D \simeq 18,6 \AA$, $E \simeq 26 \AA$ et $F \simeq 33 \AA$.

- pour $\mathrm{Ca}_{11} \mathrm{Fe}_{10} \mathrm{TiO}_{28}(n=2,20$ ou $y=0,45)$, deux microdomaines accolés relatifs aux termes $n=2$ et $n=2,50$ (figure 10).

Une observation analogue a été faite pour $\mathrm{Ca}_{7} \mathrm{Fe}_{4} \mathrm{Ti}_{3} \mathrm{O}_{19}(n=3,5 ; y \simeq 0,29)$.

Il apparait donc que, dans ce domaine de compositions $(y \geqslant 0,25,2 \leqslant n \leqslant 4)$, l'augmentation de $n$ s'effectue par l'addition progressive de feuillets d'octaèdres perpendiculairement à $O y$, phénomène qui explique l'évolution régulière du paramètre $\bar{a}_{y}$ (figure 3).

Lorsque le taux en lacunes diminue, $y<0,25$, $n>4$, les diagrammes de diffraction électronique

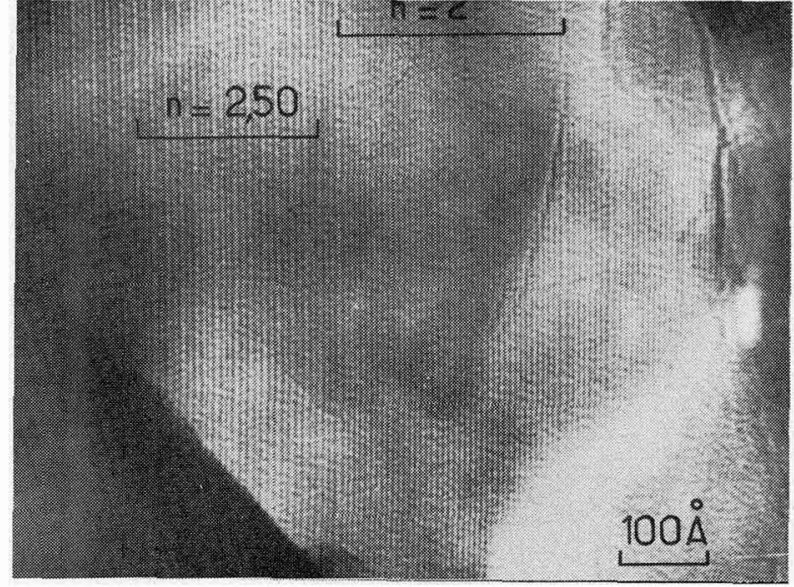

Fig, 10. - Image microscopique de réseaú de $\mathrm{Ca}_{11} \mathrm{Fe}_{10} \mathrm{TiO}_{28}$ $(n=2,20 ; y=0,45)$.

laissent apparaître une symétrie cubique. Aucun ordre des lacunes suivant des rangées [101] et des plans $(0 k 0)$ ne semble plus se manifester comme le laissait d'ailleurs prévoir l'étude par diffraction X.

Toutefois l'examen de

$$
\mathrm{Ca}_{5} \mathrm{Fe}_{2} \mathrm{Ti}_{3} \mathrm{O}_{14}(n=5, y=0,20)
$$

révèle la présence de quelques microdomaines où se manifeste un début d'ordre lacunaire (figure 11).

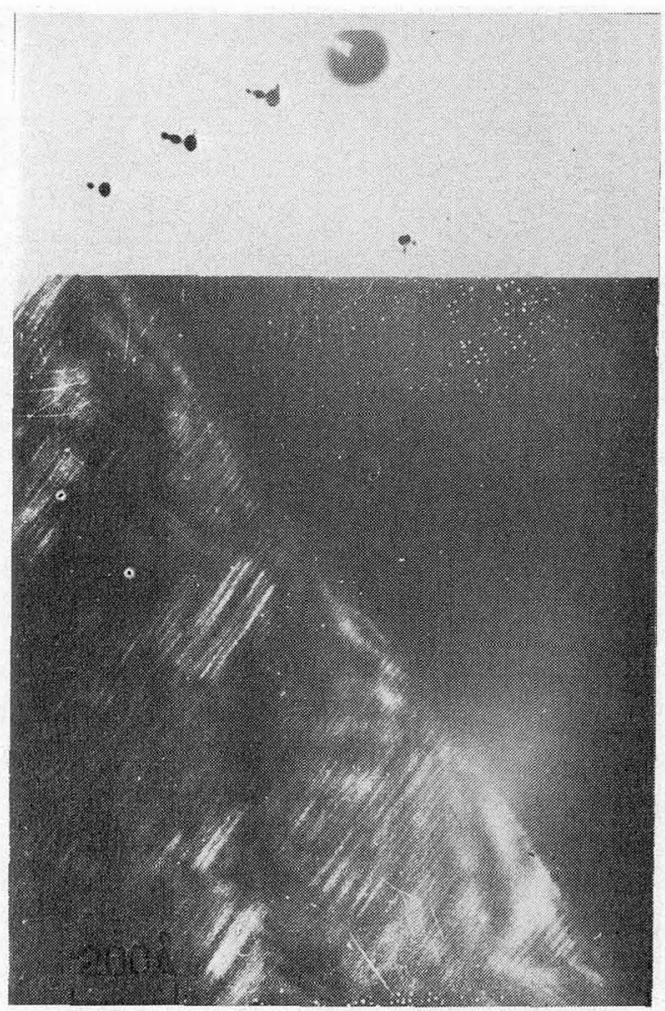

Fig. 11. - Diagramme de diffraction électronique et image de réseau de $\mathrm{Ca}_{5} \mathrm{Fe}_{2} \mathrm{Ti}_{3} \mathrm{O}_{14}(n=5, y=0,20)$. 
Spectroscopie Mössbauer : résultats expérimentaux. - L'étude par résonance Mössbauer du domaine ordonné $(y \geqslant 0,25)$ a montré que le fer trivalent occupait effectivement des sites octaédriques et tétraédriques en nombre égal. Une phase de composition $y$ peut donc s'écrire formellement :

$$
\mathrm{Ca}\left(\mathrm{Ti}_{1-2 y} \mathrm{Fe}_{y}\right)_{0}\left(\mathrm{Fe}_{y}\right)_{\mathrm{T}} \mathrm{O}_{3-y} .
$$

A titre d'exemple, la figure 12 représente le spectre obtenu à $4,2 \mathrm{~K}$ pour

$$
\mathrm{Ca}_{3} \mathrm{Fe}_{2} \mathrm{TiO}_{8}\left(y=1 / 3, \mathrm{Ca}\left(\mathrm{Fe}_{2 / 3} \mathrm{Ti}_{1 / 3}\right) \mathrm{O}_{2,67}\right) \text {. }
$$

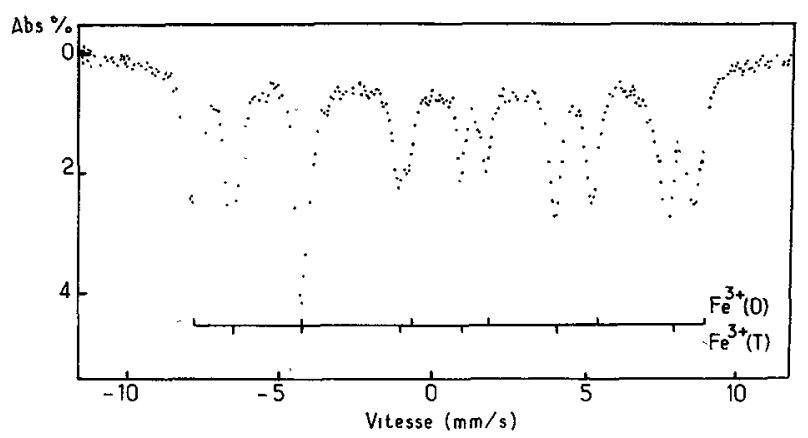

Fig. 12. - Spectre de résonance Mössbauer à $4,2 \mathrm{~K}$ pour $\mathrm{Ca}_{3} \mathrm{Fe}_{2} \mathrm{TiO}_{8}(n=3, y=1 / 3)\left({ }^{57} \mathrm{Co}(\mathrm{Rh})\right)$.

Les déplacements chimiques calculés sont caractéristiques du fer trivalent en sites octaédriques et tétraédriques. Par ailleurs le rapport entre les aires des raies relatives à ces deux sites est égal à 1 .

Des résultats analogues ont été obtenus pour d'autres compositions de ce domaine; ils permettent de conclure que le titane occupe exclusivement les sites octaédriques, sites habituels pour celui-ci.

Pour les compositions correspondant à $y<0,25$, nous avons vu par diffraction $\mathrm{X}$ et microscopie électrónique que les lacunes anioniques semblaient désordonnées.

Les spectres de résonance Mössbauer obtenus à température ambiante pour $y=0,20 ; 0,05$ et 0,01 révèlent la présence de fer en sites octaédriques mais aussi en sites tétraédriques même pour de très faibles taux en lacunes. De plus le rapport $\mathrm{Fe}_{(\mathrm{T})} / \mathrm{Fe}_{(\mathrm{O})}$ diminue lorsque $y$ décroît (figure 13) $\left({ }^{1}\right.$ ).

Conclusions générales. - Pour étudier les phénomènes d'ordre-désordre dans les perovskites lacunaires $\mathrm{CaTi}_{1-2 y} \mathrm{Fe}_{2 y} \mathrm{O}_{3-y}(0 \leqslant y \leqslant 0,50)$, trois techniques d'investigations ont été nécessaires : la diffraction $\mathrm{X}$, la microscopie électronique et la spectroscopie Mössbauer. Elles ont permis de mettre en évidence une tendance des défauts anioniques à constituer autour de certains éléments, le fer en particulier, des tétraèdres oxygénés.

(1) Ménil, F. et Grenier, J. C., Communication privée.
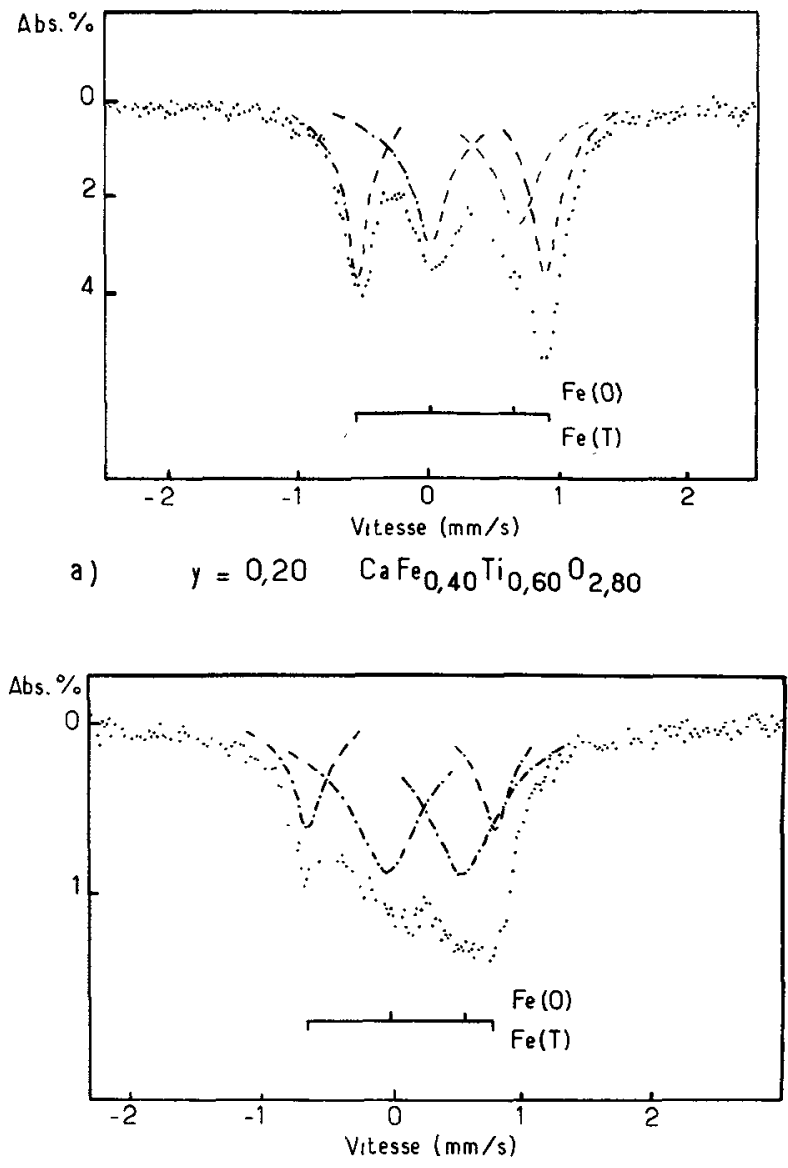

b) $\quad y=0,05 \quad \mathrm{CaFe}_{0,1} \mathrm{Ti}_{0,9} \mathrm{O}_{2,95}$

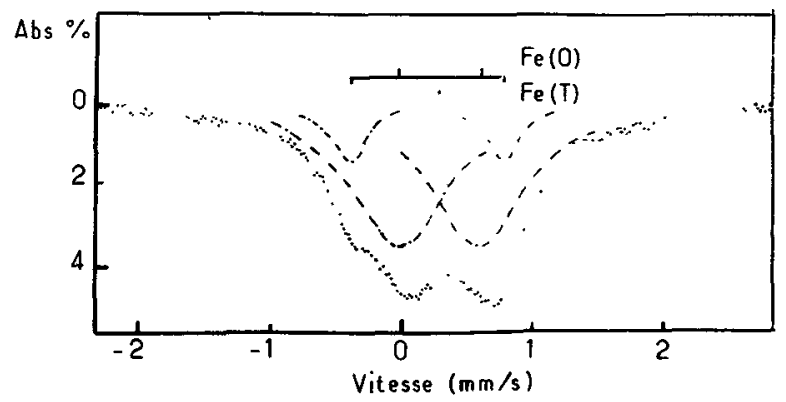

c) $y=0,01 \quad \mathrm{CaFe}_{0.02} \mathrm{Ti}_{0,98} \mathrm{O}_{2,99}$

FIG. 13. - Spectres de résonance Mössbauer à $295 \mathrm{~K}$ pour les phases $\mathrm{CaTi}_{1-2 y} \mathrm{Fe}_{2 y} \mathrm{O}_{3-y}:(a) y=0,20 ;(b) y=0,05 ;(c) y=0,01$.

Pour de faibles taux en lacunes $(y<0,25)$, ceux-ci sont statistiquement distribués au sein du réseau, la symétrie pseudocubique de la perovskite est conservée. La formation de ces tétraèdres implique l'association d'au moins deux lacunes sur un octaèdre initial ; leur nombre augmente lorsque $y$ croît.

Pour des taux plus importants $(y \geqslant 0,25)$, ils s'ordonnent en files dans des plans $(0 k 0)$ conduisant à la formation de nouvelles structures cristallines que décrit notre modèle. La non-stœchiométrie de ces 
phases est originale dans la mesure où on n'observe pas une compression de la structure par un mécanisme de cisaillement. Elle est assurée en revanche par un ordre des lacunes et une succession perpendiculairement à l'axe $O y$ de plans d'octaèdres et de plans de tétraèdres. Ces phases constituent en fait une intercroissance syntaxique (intergrowth) ordonnée ou désordonnée des termes extrêmes $n=2$ et $n=\infty$.

Il semble difficile pour le moment de généraliser ces résultats à l'ensemble des phases $\mathrm{AMO}_{3-y}$ dans la mesure où l'élément $M$ n'a pas nécessairement tendance à prendre un environnement tétraédrique.

\section{Bibliographie}

[1] Collongues, R., La non-stoechiométrie (Masson, Paris) 1971.

[2] Le RoY-Eyring et O'KeEFFe, M., The Chemistry of Extended Defects in Non-Metallic Solids (North Holland, Amsterdam) 1970.

[3] Tofield, B. C., Greaves, C. et Fender, B. E. F., Mat. Res. Bull. 10 (1975) 737.
[4] Grenier, J. C., Darriet, J., Pouchard, M. el HagenmulLTR, P., Mat. Res. Bull. 11 (1976) 1219.

[5] Shannon, R. D. et Prewitt, C. T., Acta Crystallogr. B 25 (1969) 928.

[6] Grenier, J. C., Schiffmacher, G., Caro, P., Pouchard, M. et Hagenmuller, P., J. Sol. State Chem. 20 (1977) 365. 